\title{
Solid Lipid Nanoparticles of Cyclosporine for the Treatment of Skin Disease
}

\author{
Ramila Prajapati ${ }^{*}$, Dhavalkumar Patel ${ }^{2}$ and Jayvadan Patel ${ }^{1}$ \\ ${ }^{1}$ Faculty of Pharmacy, Sankalchand Patel University, Visnagar, Gujarat, India \\ ${ }^{2}$ Leading Pharma LLC, New Jersey, USA \\ *Corresponding Author: Ramila Prajapati, Faculty of Pharmacy, Sankalchand Patel \\ University, Visnagar, Gujarat, India.
}

Received: February 24, 2021

Published: March 20, 2021

(C) All rights are reserved by Ramila

Prajapati., et al.

\section{Abstract}

Cyclosporine is one of the shows potential drugs and employ for the treatment of variety of skin diseases like Psoriasis. However, High blood pressure, Swollen or inflamed gums, and other common side effects are tremors, restlessness, stomach upset, nausea, cramps, diarrhea, headache limit its clinical applications.

The reported work pivot on the preparation of solid lipid nanoparticles (SLN) using cyclosporine drug, for the enhancement of their penetration efficacy in the skin. Solid lipid nanoparticles (SLNs) of the drug by the using of many type of lipids, like palmitic acid, glyceryl behenat, cetyl palmitate and glyceryl monostearate. Prepared SLN was validated regarding particle size, zeta potential, percentage entrapment efficiency (EE). In-vitro dermatokinetics and drug efficacy evaluated respectively by tape stripping method and using HaCaT cell lines. The particle size of nanoformulation was ranging less than $350 \mathrm{~nm}$ and the morphology is showing spherical. HaCaT cell lines were used for the in-vitro study that showed higher uptake and efficacy with decrease cell viability for SLNs.

Keywords: Cyclosporine; Fatty Acids; SLN; HaCaT Cell Line

\section{Introduction}

Psoriasis is one of the more widespread forms of unending dermal disease in the world. In fact, at hand is no good remedy for psoriasis but there are many special treatment way to reduce the harshness of symptoms. This disease is an auto-immune of skin with frequent portion of hyperkeratosis and inflammation connecting abnormal commencement of T cells, their relocation into the skin and final aggregation [1].

The main remedy therapy in the treatment psoriasis presuppose dermal or topical drug delivery remaining to its benefit of least systemic absorption and consequently with minor toxicity. None of topical drug category is ideal for the management of psoriasis; apart from the main difficulty in combinational drug therapy is the trouble in administration; so, requirements arise to develop a nanoformulation for the improved pharmacokinetic effect of the drug $[2,3]$.

Cyclosporine (CsA) present anti psoriatic activity outstanding, due to shyness of $\mathrm{T}$ cells and its immune responses which are hyper active in psoriasis. It also inhibits IL-2 production which in turn reduces the production of secondary cytokines (TNF $\alpha$, IL-17, IL-23) which are likely to be the mechanisms of keratinocyte production [4].

Cyclosporine A (BCS Class II, Biopharmaceutical Classification System) is a fat soluble, hydrophobic polypeptide metabolite of fungus Beauveria nivea (formerly Tolypocladium inflatum Gams) [5]. It is a hydrophobic cyclic peptide built from non-mammalian amino acids with low oral bioavailability, which is one of first line 
immunosuppressive drugs used to prevent transplant rejection and to treat autoimmune diseases $[6,7]$.

\section{Materials and Methods}

Drug profile

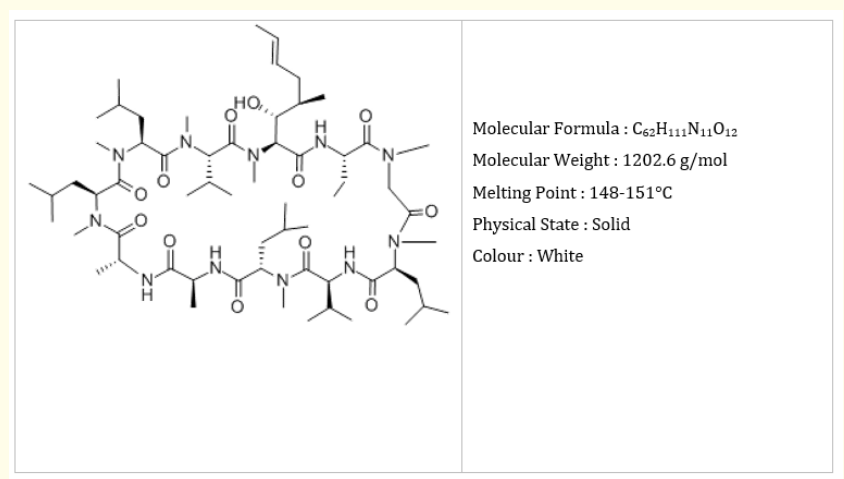

Figure 1: Molecular structure of cyclosporine (CsA).

Solubility study of cyclosporine in different oils, surfactants and co-surfactants:

The solubility study of Cyclosporine was studied in different oils, surfactants and co-surfactants, including: Carpryol 90, Lauroglyol 90 , Peceol, Labrafac Lipophile, Span 80, PEG-400, Labrafil, PEG35 Castor oil, PEG-40 Hydrogenate Castor Oil, Propylene Glycol, Labrasol $[8,9]$. Cyclosporine A was mixed in $10 \mathrm{ml}$ vial with such amounts of each of the above solvents in order to produce supersaturated solutions. The mixtures were shaken at constant vibration under ambient temperature for 2 days for equilibrium. The obtained suspensions were centrifuged at $5000 \mathrm{rpm}$ for 20 minutes. Then an accurately weighed quantity of supernatant was further diluted with methanol and analyzed using an HPLC method for its drug content.

Preparation of cyclosporine solid lipid nanoparticles (SLNs)

The Cyclosporine was dissolved in the hot molten lipid matrix (palmitic acid, glyceryl behenat, cetyl palmitate and glyceryl monostearate) which is then dispersed in the hot aqueous surfactant solution using high speed stirrer (e.g. ultra turrax) to form a coarse pre emulsion which is then homogenized using high pressure homogenizer at appropriate pressures with sufficient number of cycles (homogenization method). After this the o/w nanoemulsion is cooled to room temperature followed by lipid crystallization which leads to the formation of solid lipid nanoparticles $[10,11]$.

\begin{tabular}{|l|c|}
\hline Oils, surfactants and co-surfactants & Solubility(mg/g) \\
\hline Carpryol 90 & $496.0 \pm 1.3$ \\
\hline Lauroglyol 90 & $469.2 \pm 2.2$ \\
\hline Peceol & $228.2 \pm 2.8$ \\
\hline Labrafac Lipophile & $302.4 \pm 2.4$ \\
\hline Span 80 & $152.4 \pm 2.2$ \\
\hline PEG-400 & $287.6 \pm 3.2$ \\
\hline PEG35 Castor oil & $161.8 \pm 1.2$ \\
\hline Labrasol & $152.2 \pm 2.1$ \\
\hline PEG-40 & $172.2 \pm 3.8$ \\
\hline Hydrogenate Castor Oil & $205.2 \pm 1.3$ \\
\hline Propylene Glycol & $185.4 \pm 1.2$ \\
\hline
\end{tabular}

Table 1: Solubility of cyclosporine A in different oils, surfactants and co-surfactants.

\section{Characterization of SLNS}

The surface morphological examinations of SLNs were carried out with scanning electron microscopy (Model JSM 5610 LV SEM, Japan).

Laser diffraction particle size analyzer (Zetasizer Nano S90, Malvern, UK) were used for determination of the particle size as well as particle size distribution of the SLNs.

\section{Determination of entrapment efficiency (EE)}

Cyclosporine in the SLNs was analyzed by dissolving the SLNs in $0.5 \%$ Polysorbate-20 in PBS (pH 5.8). The concentration of SLNs in $0.5 \%$ Polysorbate-20 in PBS ( $\mathrm{pH} 5.8$ ) was $05 \mathrm{mg} / \mathrm{ml}$. The mixture was stirred continuously overnight at room temperature. Solutions were analyzed by above-mentioned high-performance liquid chromatograph (HPLC) at a wavelength of $212 \mathrm{~nm}$ for cyclosporine A content (Shimadzu LC10AD) with suitable dilutions. Percent EE was calculated using following equation $[12,13]$.

$\% \mathrm{EE}=$ (Amount of drug entrapped/Total amount of drug taken) $\times 100$

Stability Study of SLNs

The changes in the particle size and EE of SLNs were widely used as indicators of storage stability. Optimized batch of SLNs were stored at $4^{\circ} \mathrm{C}$ and $25^{\circ} \mathrm{C}$ for 3 months. The physical stability of the samples were evaluated on 0 days, $3^{\text {rd }}$ month and $6^{\text {th }}$ month for any change in particle size, PDI and \% EE $[14,15]$. 


\section{Drug release kinetics}

Dialysis methods are an appealing alternative to investigate release of drug from in-situ depot forming system and other formulations including implants. The in vitro release studies of Cyclosporine from the formulation were studied using the dialysis Cassettes (10000 MWCO, Thermo Scientific). A dialysis Cassettes previously soaked overnight in the diffusion medium and a defined amount of drug-loaded nanoparticles containing $0.1 \mathrm{mg}$ Cyclosporine was accurately inserted using syringe into dialysis Cassettes. The dialysis Cassettes was suspended in a beaker containing $100 \mathrm{~mL}$ of PBS (pH 5.8) at $(37 \pm 0.5)^{\circ} \mathrm{C}$. This assembly was kept on magnetic stirrer at $50 \mathrm{rpm}$. At fixed intervals $(2.25 \mathrm{~h}$, 2.5 h, 2.75 h, 3 h, 3.5 h, 4 h, 6 h, 8 h, 12 h and 24 h) 3 mL samples were withdrawn over $24 \mathrm{hrs}$ and were refilled with fresh dissolution medium. Withdrawn samples were analyzed by above-mentioned high-performance liquid chromatograph (HPLC) at a wavelength of $212 \mathrm{~nm}$ for cyclosporine A content (Shimadzu LC10AD). In-vitro drug release data were fitted to zero-order, first-order, Higuchi equation, Korsemeyer-Peppas equations and hixon crowel model, as per in Table 2, and regression analysis was performed to find the best fitted one [16-22].

\begin{tabular}{|l|c|c|c|}
\hline S. No. & Model & Graph & Equations \\
\hline 1. & Zero order model & Time versus $\%$ Drug release & $\mathrm{Q}_{0}-\mathrm{Q}_{\mathrm{t}=} \mathrm{K}_{0} \mathrm{t}$ \\
\hline 2. & First order model & Time versus Log $\%$ Drug unreleased & $\log \mathrm{C}=\log \mathrm{C}_{0}-\mathrm{K}_{\mathrm{t} / 2.303}$ \\
\hline 3. & Higuchi model & Square root of time versus cummulative & $\mathrm{F}_{1}=\mathrm{Q}=\mathrm{A} \sqrt{D(2 C-C S) C S t}$ \\
\hline 4. & $\begin{array}{c}\text { Korsmeyer peppas } \\
\text { model }\end{array}$ & Time versus log \%cumulative drug release & $\mathrm{M}_{\mathrm{t}} / \mathrm{M}_{\infty}=\mathrm{Kt}^{\mathrm{n}}$ \\
\hline
\end{tabular}

Table 2: Various Kinetic Models for Drug Release [23,24].

\section{In-vitro HaCaT cell line studies [25-27]}

The in-vitro efficacy studies were carried out using HaCaT cell lines, as they mimic the hyperproliferative and impaired differentiation of impaired psoriatic epidermal keratinocytes. HaCaT cells were placed in 24 well plates at a density of 4 x 105 cells/well and were allowed to grow and attach for $24 \mathrm{hr}$ before treatment with free drug mixture, blank formulation and formulation loaded with Cyclosporine A. After UV A radiation, CoQ10 were added to wells. Before MTT test, each well was washed with PBS, 7601 fresh medium and $0.5 \%$ MTT 40 were added and then were incubated for $3 \mathrm{hr}$. Finally, DMSO was added to dissolve purple formazan and absorption was determined by the absorption in an enzyme linked immunosorbent assay plate reader at $490 \mathrm{~nm}$ and reference wavelength at $630 \mathrm{~nm}$. The results were expressed in terms of \% growth inhibition. These cell line studies are used to assess the in vitro anti psoriatic activity as these mimic the hyperproliferation and impaired characterization of epidermal keratinocytes. These cell lines efficiently predict the uptake of formulations by the cells.

\section{Results}

Standard plot of cyclosporine in ethanol

In the studied range of $1-8 \mu \mathrm{g} / \mathrm{mL}$ of DTX-PL in ethanol, the observed $\lambda$ max was $214 \mathrm{~nm}$. The standard calibration curve of cyclosporine was obtained by plotting Absorbance vs. Concentration.

\begin{tabular}{|l|c|c|}
\hline S. No. & $\begin{array}{c}\text { Concentration } \\
(\mu \mathrm{g} / \mathbf{m l})\end{array}$ & $\begin{array}{c}\text { Mean Absorbance } \\
( \pm \mathbf{)} \text { SD }\end{array}$ \\
\hline 1. & 1 & $0.092 \pm 0.01$ \\
\hline 2. & 2 & $0.198 \pm 0.02$ \\
\hline 3. & 3 & $0.295 \pm 0.01$ \\
\hline 4. & 4 & $0.402 \pm 0.03$ \\
\hline 5. & 5 & $0.565 \pm 0.04$ \\
\hline 6. & 6 & $0.620 \pm 0.03$ \\
\hline 7. & 7 & $0.789 \pm 0.04$ \\
\hline 8. & 8 & $0.878 \pm 0.02$ \\
\hline
\end{tabular}

Table 3: Standard plot of cyclosporine in ethanol. 


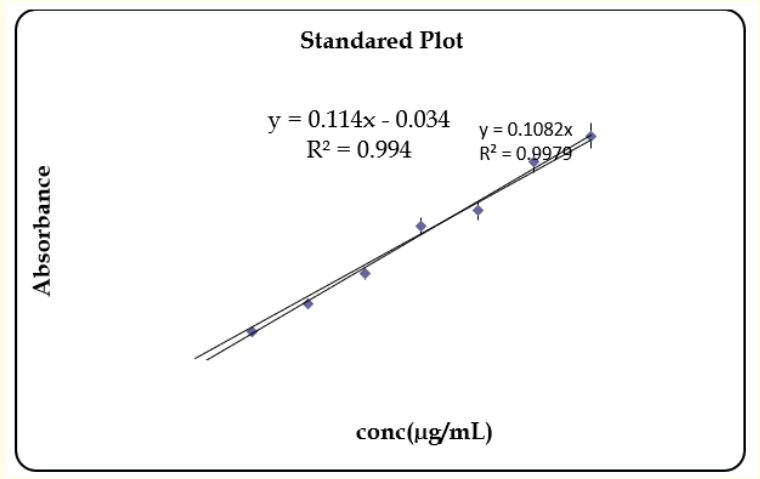

Figure 2: Calibration curve of Cyclosporine in Ethanol.

\section{Morphology of SLNs}

Scanning electron microscopy (SEM) was utilized to evaluate the morphology of the SLNS. Figure 3 display SEM photographs for Cyclosporine loaded SLNs. SEM images of solid lipid nanoparticles clearly indicated the interaction of drug with respective carrier material and concluded the incorporation of drug in matrix material [28-30].

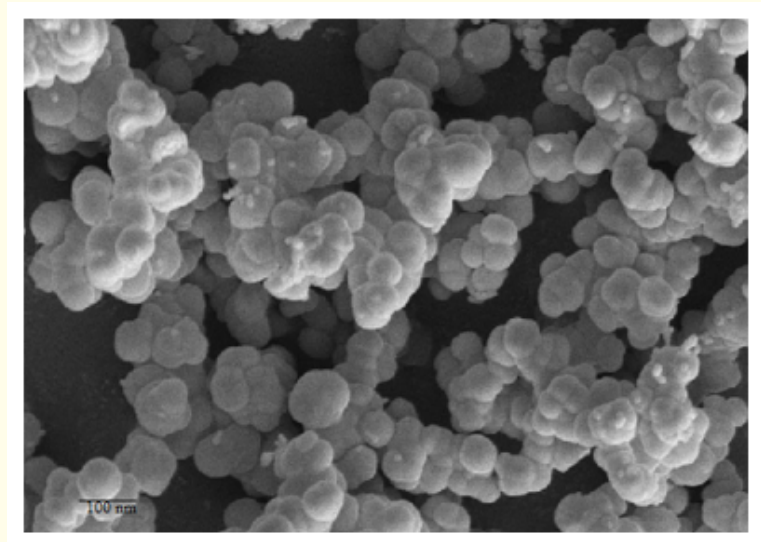

Figure 3: SEM photographs for Cyclosporine loaded SLNs.

Particle size, surface charge analysis entrapment efficacy (EE)

Table 4 represent the data of the particle size, zeta potential and PDI along with drug entrapment efficacy for all the developed SLNs loaded with cyclosporine, viz. palmitic acid derived SLNs (Pal-SLN), cetyl palmitate-derived SLNs (Cpal-SLN), glycerol monostearatebased SLNs (Gcms-SLN) and glyceryl behenat -based SLNs (GcbSLN). Model of size was observed as Gcms-SLN > Pal-SLN > CpalSLN > Gcb-SLN [30].

\begin{tabular}{|l|c|c|c|c|c|}
\hline S. No. & $\begin{array}{c}\text { Name of } \\
\text { Sample }\end{array}$ & $\begin{array}{c}\text { Particle } \\
\text { size } \\
\text { (nm) }\end{array}$ & PDI & $\begin{array}{c}\text { Zeta } \\
\text { potential } \\
\text { (mV) }\end{array}$ & \% EE \\
\hline 1. & Pal-SLN & $\begin{array}{c}239.80 \pm \\
12.01\end{array}$ & 0.302 & -14.9 & $89.16 \pm 0.25$ \\
\hline 2. & Cpal-SLN & $\begin{array}{c}183.78 \pm \\
15.84\end{array}$ & 0.290 & -21.9 & $92.09 \pm 0.78$ \\
\hline 3. & Gcms-SLN & $\begin{array}{c}321.00 \pm \\
14.89\end{array}$ & 0.367 & -29.3 & $94.70 \pm 0.17$ \\
\hline 4. & Gcb-SLN & $\begin{array}{c}152.07 \pm \\
10.52\end{array}$ & 0.367 & -8.9 & $91.38 \pm 0.53$ \\
\hline
\end{tabular}

Table 4: The obtained data pertaining to particle size, surface charge and drug entrapment studied SLNs.

The EE of the CAs in all the SLNs was near by $90 \%$. The PDI values of developed SLNs confirmed the reliability of the obtained particle size distribution range $(\leq 4)$.

\section{In-vitro Drug Release Studies and Release Kinetics}

The findings indicate that the developed SLNs own drug release controlling latent, which is the most preferred characteristic of nanocarrier-based drug delivery. The results of drug release have been shown in figure 4 .

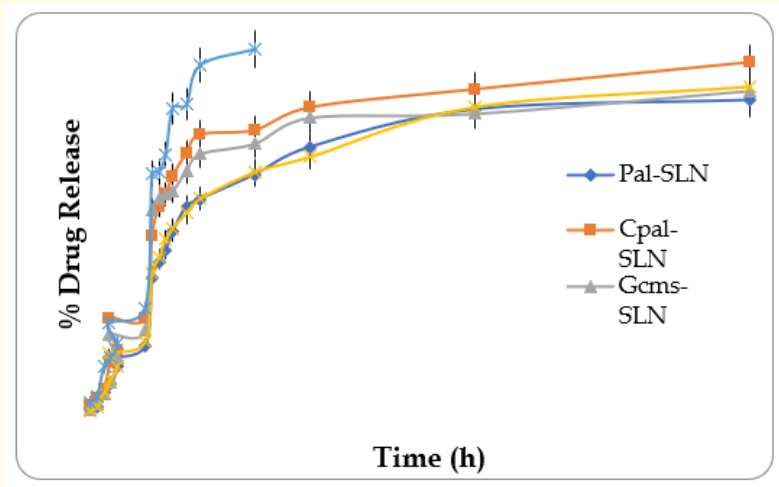

Figure 4: Drug release plot of CAs from the developed SLNs in PBS $\mathrm{p}^{\mathrm{H}} 5.8$ for $24 \mathrm{~h}$. 
The drug release data were showing to drug release kinetics employing the mostly working models like zero-order, first-order,
Higuchi and Korsmeyer-Peppas. The evolution data of the modeling have been represented in table 5 .

\begin{tabular}{|c|c|c|c|c|c|c|c|c|c|c|}
\hline \multirow{2}{*}{$\begin{array}{l}\text { Kinetic } \\
\text { Model }\end{array}$} & \multicolumn{2}{|c|}{ Plain CAs } & \multicolumn{2}{|c|}{ Pal-SLN } & \multicolumn{2}{|c|}{ Cpal-SLN } & \multicolumn{2}{|c|}{ Gcms-SLN } & \multicolumn{2}{|r|}{ Gcb-SLN } \\
\hline & $\mathbf{R}^{2}$ & Equation & $\mathbf{R}^{2}$ & Equation & $\mathbf{R}^{2}$ & Equation & $\mathbf{R}^{2}$ & Equation & $\mathbf{R}^{2}$ & Equation \\
\hline Zero-order & 0.872 & $18.33 x+7.346$ & 0.899 & $11.99 x+4.145$ & 0.867 & $14.49 x+7.591$ & 0.83 & $14.21 x+6.427$ & 0.9 & $12.05 x+4.602$ \\
\hline First-order & 0.779 & $0.223 x+1.041$ & 0.796 & $0.224 x+0.843$ & 0.768 & $0.215 x+0.984$ & 0.749 & $0.235 x+0.895$ & 0.782 & $0.226 x+0.848$ \\
\hline Higuchi & 0.863 & $46.44 x-14.74$ & 0.876 & $30.14 x-10.00$ & 0.879 & $37.17 x-10.47$ & 0.851 & $36.67 x-11.58$ & 0.886 & $30.47 x-9.847$ \\
\hline $\begin{array}{l}\text { Korsmeyer- } \\
\text { Peppas }\end{array}$ & 0.802 & $0.984 x+1.305$ & 0.831 & $1.000 x+1.107$ & 0.813 & $0.967 x+1.236$ & 0.801 & $1.060 x+1.17$ & 0.814 & $1.003 x+1.115$ \\
\hline
\end{tabular}

Table 5: Drug release kinetics of CAs and its solid lipid nanoparticles (SLNs).

\section{In-vitro HaCaT cell line studies}

It was observed from the figure 5 , that $50 \%$ of the cells were inhibited by Cyclosporine SLNs when dose of $50 \mu \mathrm{g} / \mathrm{mL}$ was given and cell viability decreased progressively as the dose was increased. On the other hand free Cyclosporine were not able to inhibit $50 \%$ of the growth when highest dose of $100 \mu \mathrm{g} / \mathrm{ml}$ was given, thereby proving the fact that Cyclosporine SLNs had better effect on cell growth as compared to the free drug which was in turn due to higher uptake by the cells. It was also observed that cell viability was not affected with blank SLNs, which proved that there was no effect of surfactant concentration and surfactant type on cell viability causing no cell death $[31,32]$.

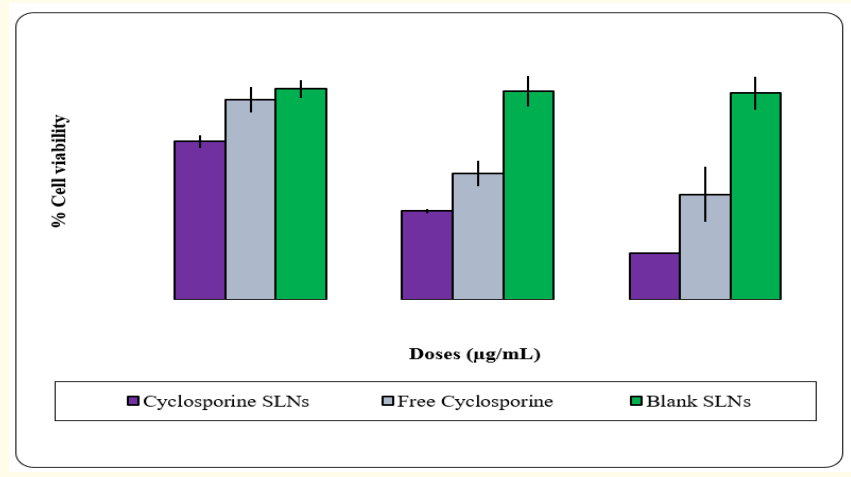

Figure 5: Comparative evaluation of \% Cell viability of free drug, blank SLNs, and Cyclosporine SLNs. Each value is expressed as mean \pm S.D $(n=3)$.

\section{Stability studies}

To picture the stability prospective of the developed SLNs, was performed shown in table 6. It was establish that there was less increase in the particle size which can be credited to the development of slight aggregates. But there was no important effect in the entrapment efficacy of the SLNs. This guaranteed that the prepared SLNs were appropriate to be stored at the room temperature, at lease for 6 months.

\begin{tabular}{|l|c|c|c|c|}
\hline \multicolumn{5}{|c|}{ Pal-SLN } \\
\hline Months & $\begin{array}{c}\text { Particle size } \\
\text { (nm) }\end{array}$ & $\begin{array}{c}\text { Zeta Potential } \\
\text { (mV) }\end{array}$ & PDI & \% EE \\
\hline Initial & $239.80 \pm 12.01$ & -14.9 & 0.302 & $89.16 \pm 0.25$ \\
\hline 3 & $245.05 \pm 11.01$ & -13.7 & 0.309 & $86.18 \pm 0.21$ \\
\hline 6 & $256.60 \pm 13.01$ & -15.8 & 0.323 & $83.78 \pm 0.19$ \\
\hline Cpal-SLN & \multicolumn{5}{|c|}{} \\
\hline Initial & $183.78 \pm 15.84$ & -21.9 & 0.290 & $92.09 \pm 0.78$ \\
\hline 3 & $193.89 \pm 14.87$ & -20.8 & 0.298 & $95.00 \pm 0.68$ \\
\hline 6 & $198.78 \pm 16.89$ & -20.8 & 0.311 & $89.04 \pm 0.58$ \\
\hline Gcms-SLN & $321.00 \pm 14.89$ & -29.3 & 0.367 & $94.70 \pm 0.17$ \\
\hline Initial & $351.00 \pm 13.89$ & -36.3 & 0.378 & $91.60 \pm 0.15$ \\
\hline 3 & $371.60 \pm 17.89$ & -36.9 & 0.379 & $84.70 \pm 0.20$ \\
\hline 6 & $152.07 \pm 10.52$ & -8.90 & 0.367 & $91.38 \pm 0.53$ \\
\hline Gcb-SLN & $162.17 \pm 09.52$ & -9.90 & 0.389 & $87.13 \pm 0.57$ \\
\hline Initial & $188.57 \pm 08.52$ & -11.03 & 0.403 & $84.45 \pm 0.35$ \\
\hline 3 & & &
\end{tabular}

Table 6: Stability studies observation of the developed SLNs. 


\section{Conclusion}

The existing studies effectively embarked upon the preparation and characterization of solid lipid nanoparticles of Cyclosporine. The appearance of studies novel drug delivery systems and its added development has concerned the curiosity of researchers in psoriasis. The lipid-based nano-carriers resulted in approving interactions with the cell lines, and found to be enhanced tolerated. This study not only begains upon with the intend space and the perfect formulation, but also well-known the cause and result relationship along-with mechanistic role SLN characteristics. Thus, it can be concluded that SLNs offer a better option for the delivery of more side effect having and less skin penetrate molecules like Cyclosporin.

\section{Acknowledgements}

The authors wish to acknowledge Faculty of Pharmacy, Sankalchand Patel University, Visnagar, Gujarat, India for providing the working platform. Authors also want to express their thanks to Chairman, Sankalchand Patel University for providing necessary facilities and infrastructure.

\section{Declaration of Competing Interest}

The authors declare that they have no known competing financial interests or personal relationships that could have appeared to influence the work reported in this paper.

\section{Bibliography}

1. Chandra A., et al. "Genetic and epigenetic basis of psoriasis pathogenesis". Molecular Immunology 64 (2015): 313-323.

2. Griffiths CEM and Barker JNWN. "Pathogenesis and clinical features of psoriasis". The Lancet 370 (2007): 263-271.

3. Lin Y-K., et al. "Combination of calcipotriol and methotrexate in nanostructured lipid carriers for topical delivery". International Journal of Nanomedicine 5 (2010): 117-128.

4. Kim ST., et al. "Topical administration of cyclosporin A in a solid lipid nanoparticle formulation". International Journal of Pharmacy and Pharmaceutical Sciences 64 (20009): 510-514.

5. Austad J., et al. "Clobetasol propionate followed by calcipotriol is superior to calcipotriol alone in topical treatment of psoriasis". Journal of the European Academy of Dermatology and Venereology 11 (1998): 19-24.
6. Chaturvedi SP., et al. "A Review on Disease Management and Drug Delivery Aspects in Psoriasis". Current Trends in Technology and Sciences 1 (2012): 122-125.

7. Rahman M., et al. "Nanomedicine-based drug targeting for psoriasis: potentials and emerging trends in nanoscale pharmacotherapy". Expert Opinion on Drug Delivery 12 (2015): 635-652.

8. Souto E., et al. "Lipid-based colloidal systems (nanoparticles, microemulsions) for drug delivery to the skin: materials and end-product formulations". Journal of Drug Delivery Science and Technology 21 (2011): 43-54.

9. Müller RH., et al. "Solid lipid nanoparticles (SLN) and nanostructured lipid carriers (NLC) in cosmetic and dermatological preparations". Advanced Drug Delivery Reviews 54 (2002): S131-S55.

10. Zhang Z., et al. "Polymeric nanoparticles-based topical delivery systems for the treatment of dermatological diseases". Wiley Interdisciplinary Reviews: Nanomedicine and Nanobiotechnology 5 (2013): 205-218.

11. Thapa RK and Yoo BK. "Evaluation of the effect of tacrolimus-loaded liquid crystalline nanoparticles on psoriasis-like skin inflammation". Journal of Dermatological Treatment 25 (2014): 22-25.

12. Bessar H., et al. "Functionalized gold nanoparticles for topical delivery of methotrexate for the possible treatment of psoriasis". Colloids and Surfaces B: Biointerfaces 141 (2016): 141-147.

13. Pinto MF., et al. "A new topical formulation for psoriasis: development of methotrexate-loaded nanostructured lipid carriers". International Journal of Pharmaceutics 477 (2014): 519-526.

14. Kokelj F., et al. "Calcipotriol improves the efficacy of cyclosporine in the treatment of psoriasis vulgaris". Journal of the European Academy of Dermatology and Venereology 10 (1998): 143-146.

15. Zhou L., et al. "Development of a high throughput equilibrium solubility assay using miniaturized shake-flask method in early drug discovery". Journal of Pharmaceutical Sciences 96 (2007): 3052-3071.

16. Mukherjee S., et al. "Solid lipid nanoparticles: a modern formulation approach in drug delivery system". Indian Journal of 
Pharmaceutical Sciences 71 (2009): 349.

17. Jain S., et al. "Enhanced topical delivery of cyclosporin-A using PLGA nanoparticles as carrier". Current Nanoscience 7 (2011): 524-530.

18. Chen J., et al. "Glucosamine derivative modified nanostructured lipid carriers for targeted tumor delivery". Journal of Materials Chemistry 22 (2012): 5770-5783.

19. Fry DW., et al. "Rapid separation of low molecular weight solutes from liposomes without dilution". Analytical Biochemistry 90 (1978): 809-815.

20. Raza K., et al. "Nano-lipoidal carriers of tretinoin with enhanced percutaneous absorption, photostability, biocompatibility and anti-psoriatic activity". International Journal of Pharmaceutics 456 (2013): 65-72.

21. Feng X., et al. "Lipid-modified conjugated polymer nanoparticles for cell imaging and transfection". Journal of Materials Chemistry 20 (2010): 1312-1316.

22. Harde H., et al. "Development of a topical adapalenesolid lipid nanoparticle loaded gel with enhanced efficacy and improved skin tolerability". RSC Advances 5 (2015): 43917-43929.

23. Jain S., et al. "Enhanced dermal delivery of acyclovir using solid lipid nanoparticles". Drug Delivery and Translational Research 1 (2011): 395-406.

24. Baroli B., et al. "Microemulsions for topical delivery of 8-methoxsalen". Journal of Control Release 69 (2000): 209-218.

25. Breitkreutz D., et al. "Epidermal morphogenesis and keratin expression in c-Ha-ras-transfected tumorigenic clones of the human HaCaT cell line". Cancer Research 51 (1994): 44024409.

26. Rückert R., et al. "Inhibition of keratinocyte apoptosis by IL15: a new parameter in the pathogenesis of psoriasis?" Journal of Immunology 165 (2000): 2240-2250.

27. Patil-Gadhe A., et al. "Montelukast-loaded nanostructured lipid carriers: part II pulmonary drug delivery and in vitro-in vivo aerosol performance". European Journal of Pharmaceutics and Biopharmaceutics 88 (2014): 169-177.
28. Mehnert W., et al. "Solid lipid nanoparticles: production, characterization and applications". Advanced Drug Delivery Reviews 47 (2001): 165-196.

29. Schwarz C., et al. "Solid lipid nanoparticles (SLN) for controlled drug delivery. I. Production, characterization and sterilization". Journal of Control Release 30 (1994): 83-96.

30. Severino P., et al. "Current state-of-art and new trends on lipid nanoparticles (SLN and NLC) for oral drug delivery". Journal of Drug Delivery 2011 (2012).

31. Testerman T., et al. "Cytokine induction by the immunomodulators imiquimod and S-27609". Journal of Leukocyte Biology 58 (1995): 365-372.

32. Jain AK., et al. "Solidified self-nanoemulsifying formulation for oral delivery of combinatorial therapeutic regimen: part I. Formulation development, statistical optimization, and in vitro characterization". Pharma Research 31 (2014): 923-945.

\section{Assets from publication with us}

- Prompt Acknowledgement after receiving the article

- Thorough Double blinded peer review

- Rapid Publication

- Issue of Publication Certificate

- High visibility of your Published work

Website: www.actascientific.com/

Submit Article: www.actascientific.com/submission.php Email us: editor@actascientific.com

Contact us: +919182824667 